Cytogenet Genome Res 1968;7:336

\title{
Authors' erratum
}

\section{Received April 15, 1968}

Cytogenet Genome Res 1967;6:420-435

The identification of the chromosomes of the E group (16-18 Denver): an autoradiographic and measurement study

\section{F. Giannelli R.M. Howlett}

Paediatric Research Unit, Guy's Hospital Medical School, London

The authors apologise to the readers for having overlooked errors made in the preparation of Figure $7 \mathrm{~b}$. A correct version of the figure is shown below. There is no change in the legend.

In addition, in Table III the first standard error should read 1.13 instead of 1.31, and for the data relating to patient 4, the mean grain counts $G n$ should read 7.75 and $\mathrm{G} t_{\mathrm{V}} 6.20$ (instead of 7.74 and 6.21 respectively). The mean grain count differences, consequently, should read from top to bottom as follows: $1.35,2.05,-0.50,-2.42$ (instead of $1.36,2.04,-0.51,-2.41$ ). The interpretation of Figure 7 and the final results presented in Table III are not affected by these corrections. Finally, the formula for the centromeric index (C.I.) shown on page 422 should read:

$$
\text { C.I. }-\frac{P}{p+q} \times 100
$$

\section{0}

- Normal Chromosome with highest grain count

O Normal Chromosome with grain count lower than that above * Normal Chromosome with grain count lower than that above ${ }^{3 / 8}$ Normal Chromosome with grain count lower than that above $\Delta$ Normal Chromosome with grain count lower than that above
Deleted Chromosome 